\title{
Questes
}

Revue pluridisciplinaire d'études médiévales

\section{La fin du Moyen Âge ou l'expression des possibles : le laboratoire nantais comme illustration des renversements sociaux dans un long $\mathrm{XV}^{\mathrm{e}}$ siècle}

\section{Aurore Leon}

\section{CpenEdition}

\section{Journals}

Édition électronique

URL : http://journals.openedition.org/questes/4317

DOI : 10.4000/questes. 4317

ISSN : 2109-9472

\section{Éditeur}

Les Amis de Questes

\section{Édition imprimée}

Date de publication : 10 juin 2016

Pagination : 81-93

ISSN : 2102-7188

\section{Référence électronique}

Aurore Leon, «La fin du Moyen Âge ou l'expression des possibles : le laboratoire nantais comme

illustration des renversements sociaux dans un long XVe siècle ", Questes [En ligne], 33 | 2016, mis en ligne le 15 juin 2016, consulté le 02 mai 2019. URL : http://journals.openedition.org/questes/4317 ; DOI : 10.4000/questes.4317 


\title{
La fin du Moyen Âge ou l'expression des possibles : le laboratoire nantais comme illustration des renversements sociaux dans un long $X V^{e}$ siècle
}

\author{
Aurore LEON \\ Université Paris-Sorbonne
}

\begin{abstract}
La ville de Nantes est petite,/
Mais elle de moult grant mérite,/

Parce qu'elle est très bien peuplée, $/$

Et de belles maisons parée ${ }^{1}$.
\end{abstract}

Ces quelques vers renvoient à la perception et la représentation de la ville de Nantes par des contemporains du XV $\mathrm{V}^{\mathrm{e}}$ siècle : les dimensions de la ville ne sont pas une limite à sa réputation. En effet, les vers précédents de ce blason insistent sur sa dimension de «ville-centre » du duché de Bretagne et sur sa situation de carrefour commercial. Cette première évocation littéraire de la ville de Nantes brosse à grands traits l'image d'une ville vivante, qui s'ouvre au-delà des frontières du duché de Bretagne, au cœur de dynamiques à la fois politiques et commerciales, grâce aux réalisations et ambitions du duc François II de Bretagne puis de sa fille Anne de Bretagne. Nantes s'insère donc dans un réseau de villes bretonnes aux frontières poreuses et en contact permanent avec le royaume de France. Cette centralité politique et économique en fait un laboratoire intéressant du passage entre Moyen Âge et époque moderne.

\footnotetext{
1 Charles Dugast-Matifeux, Nantes ancien et le pays nantais: comprenant la chronologie des seigneurs, gouverneurs, évêques et abbés, le pouillé diocésain et la topographie historique de la ville et du pays, Nantes, Morel, 1879, p. 126.
} 
Ici, plutôt que tenter de justifier notre propre indécision, face à un Moyen Âge finissant ou à une première Modernité, nous tenterons de lui surimposer l'analyse à grande échelle, afin de montrer que la fin du Moyen Âge ne s'inscrit pas dans un bornage. Il s'agit d'un moment de transition et en préciser les contours en supprimerait les caractères et les enjeux. S'intéresser à l'échelle urbaine conduit à appréhender cette «fin du Moyen Âge » au prisme de la société urbaine d'un large $\mathrm{Xv}^{\mathrm{e}}$ siècle et permet ainsi d'éviter les controverses et querelles historiographiques en restituant son temps à l'espace nantais. Dépasser la rupture entre Moyen Âge et Renaissance, c'est refuser la frontière temporelle pour mettre en valeur le temps des hommes, le temps de la ville, le temps des hommes dans leur ville.

Le laboratoire nantais se singularise du fait d'une triple centralité : cette ville est en effet le principal pôle urbain du duché de Bretagne, «capitale» de la Bretagne, et enfin lieu des grandes crises politique, dynastique et militaire bretonnes. Dans cet espace cristallisant les crises, il s'agit d'étudier la construction d'une identité urbaine ${ }^{2}$. Nous ne chercherons plus les signes de rupture; nous nous efforcerons plutôt de voir comment l'élite urbaine fait fi des crises et parvient à maintenir une stabilité, en particulier administrative, dans la ville. C'est à cet échelon d'étude que le moment de transition que constitue ce long $\mathrm{Xv}^{\mathrm{e}}$ siècle commence à s'entrevoir. Le $\mathrm{XV}^{\mathrm{e}}$ siècle se présente comme un longue passage entre une ville de Nantes bretonne, mise en concurrence avec Rennes à cause d'une histoire de la Bretagne construite autour de plusieurs comtés autonomes, et une ville de Nantes «française » au sens où elle profite de tous les acquis liés au rattachement du duché de

\footnotetext{
${ }^{2}$ En ce qui concerne l'identité urbaine, voir Walter Prevenier et Marc Boone, « Les villes des Pays-Bas méridionaux au bas Moyen Âge : identité urbaine et solidarités corporatives », Bulletin du crédit communal, $\mathrm{n}^{\circ}$ 183, 1993, p. 25-42. 
Bretagne à la France. Afin de fournir quelques éléments de réponse, l'étude à l'échelle de Nantes semble intéressante du fait du contexte particulier de l'intégration du duché de Bretagne au royaume de France.

Les articles et travaux sur une Bretagne autonome, voire «terre d'Europe » se sont multipliés dans les dernières décennies ${ }^{3}$, alors que le réseau urbain des villes bretonnes a surtout fait l'objet de grandes monographies urbaines ${ }^{4}$ ou d'articles d'historiens locaux ${ }^{5}$. Cette historiographie reste loin d'une appréhension de la ville en tant que laboratoire des rapports et réseaux sociaux. La ville médiévale est ainsi perçue par l'historiographie comme un lieu social, en tant qu'elle devient le siège d'une confrontation entre espace et pouvoir : la diversité des formes du «vivre en ville» constitue un observatoire des rapports sociaux, et les individus sont davantage traités dans leurs réseaux, qu'ils soient familiaux, commerciaux ou politiques. Il semble intéressant de rappeler les riches études d'histoire sociale concernant l'Italie, et plus particulièrement l'inscription spatiale du pouvoir et des hommes à Venise à la fin du Moyen Âge ${ }^{6}$, ou encore la place d'un groupe social en ville, étudié au prisme de plusieurs espaces urbains ${ }^{7}$. De plus, l'individu est désormais «spatialisé »: il ne s'agit plus seulement d'interroger des individus pris à part, au gré des sources, mais bien plutôt des acteurs de

3 1491. La Bretagne, terre d'Europe, dir. Jean Kerhervé et Tanguy Daniel, Brest/Quimper, Centre de Recherche Bretonne et Celtique/Société archéologique du Finistère, 1992.

4 Jean-Pierre Leguay, Un Réseau urbain au Moyen Âge, les villes du duché de Bretagne aux XV ${ }^{e}$ et $X V I^{e}$ siècles, Paris, Maloine, 1981.

${ }^{5}$ Les Mémoires de la Société d'Histoire et d'Archéologie de Nantes, ainsi que ceux de la Société d'Histoire et Archéologie de Bretagne proposent des synthèses, archéologiques notamment, intéressantes du point de vue de l'histoire micro-locale, à l'instar des contributions, fort anciennes néanmoins, de Léon Maitre.

${ }^{6}$ Élisabeth Crouzet-Pavan, Le Moyen Âge de Venise. Des eaux salés au miracle de pierres, Paris, Albin Michel, 2015.

7 Thierry Dutour, «Les nobles et la ville à la fin du Moyen Âge dans l'espace francophone », Cahiers de Recherches Médiévales et Humanistes, $\mathrm{n}^{\mathrm{0}}$ 13, 2006, p. 151-164. 
leur espace vécu. Par ailleurs, les études relatives à la noblesse bretonne se cantonnent le plus souvent à l'inscription dans une périodisation stricte ou encore qui n'intègre que fort peu les nouveaux «seigneurs » issus d'une bourgeoisie rentière ${ }^{8}$. En effet, les études sur la noblesse bretonne, principalement incarnée par sa chevalerie, se concentrent le plus souvent sur le XIII ${ }^{\mathrm{e}}$ siècle et la première moitié $\mathrm{du} \mathrm{XIV}^{\mathrm{e}}$ siècle $^{9}$, tandis que pour le $\mathrm{XV}^{\mathrm{e}}$ siècle on a surtout un questionnement sur la fidélité de la noblesse, introduit par le rattachement du duché de Bretagne au royaume de France $^{10}$. Enfin, la bourgeoisie ne constitue un objet d'études important qu'à partir du moment où la ville se dote d'une mairie, en $1564^{11}$. Il s'agit donc d'introduire l'étude de sources nantaises dans une historiographie largement favorable aux acteurs sociaux. L'étude des rapports sociaux à Nantes permet ainsi de dépasser une approche du duché du point de vue de sa structure féodale, et de montrer les interactions propres à une ville. La continuité avec un Moyen Âge féodal permet avant tout de conserver les caractéristiques politiques et structurelles de l'«État breton»: la force même de la structure administrative qui va nécessiter et forcer la mobilité sociale. La rupture entre Moyen Âge et époque moderne ne trouve ainsi pas d'illustration claire dans l'historiographie de l'histoire des villes.

De fait, il s'agira ici d'effectuer une synthèse, en se penchant non pas sur un acteur particulier, mais en faisant évoluer quelques individus dans leur ville, pour rompre l'anonymat du groupe et fournir quelques exemples de mutations sociales.

\footnotetext{
${ }^{8}$ Michel Nassiet, Noblesse et pauvreté : la petite noblesse en Bretagne, $X V^{e}-$ XVIII siècle, Rennes, PUR, coll. « Archives historiques de Bretagne », 2012.

${ }^{9}$ Frédéric Morvan, La Chevalerie bretonne et la formation de l'armée ducale, 1260 1341, Rennes, PUR, coll. « Histoire », 2009.

10 Jean Kerhervé, Noblesses de Bretagne du Moyen Âge à nos jours, Rennes, PUR/Institut culturel de Bretagne, coll. « Histoire », 1999.

${ }^{11}$ Guy Saupin, Nantes au XVII siècle. Vie politique et société urbaine, Rennes, PUR, coll. « Histoire », 1996.
} 


\section{Intégration à un nouveau groupe, intégration d'un nouveau}

\section{groupe : une esquisse des renversements sociaux}

Si la guerre de Bretagne ou l'approche des Français de la ville de Nantes sont largement évoquées dans les sources ${ }^{12}$, les quatre campagnes ${ }^{13}$ et plus particulièrement la première, conduisant au siège de Nantes, ne bouleversent ni ne paralysent l'activité municipale. Nous ne chercherons donc pas d'explication aux mobilités sociales dans l'histoire politique et militaire en tant que telle. En tenant compte d'un éventuel effet des sources, on pourra ainsi remarquer l'homogénéité d'un espace urbain imperméable aux crises.

J'examinerai les différentes manières de s'intégrer au corps de la ville, pour ces «bourgeois, habitants et manants de Nantes », qui n'ont pas encore d'institution claire au sein de laquelle se manifester. En effet, il est inutile de rechercher des signes de bouleversements dans le personnel des institutions politiques et administratives lors du rattachement au royaume de France.

L'appartenance à un groupe se manifeste à l'échelon du pouvoir urbain, et à un échelon politique plus fin, celui de l'administration ducale. Des lignées familiales servent tour à tour la ville et le duché, et profitent de cette façon de stratégies d'ascension sociale. Le cas des Lespervier est révélateur à cet égard: on trouve la mention d'un premier Lespervier, Jean, en 1443, mais c'est surtout le lignage à partir de Georges qui retient notre attention. Georges est lieutenant et garde du château de Nantes en

\footnotetext{
12 Nous retrouvons à plusieurs reprises la mention de «Français, ennemis du duc », « incursions de l'ennemi », etc., dans des documents datés de 1487 à 1489 (Nantes, Archives Municipales, BB 1, $12 \mathrm{r}^{\mathrm{o}}$; Nantes, Archives Municipales, BB 1, $15 \mathrm{r}^{\mathrm{o}}$ ); Nantes, Archives Municipales, BB 142, $7 \mathrm{r}^{\circ}$ ) qui témoignent de la situation de la ville à la veille du siège opéré par les Français.

${ }^{13}$ Campagnes menées par le roi de France en 1487, 1488, 1489, 1491.
} 
1450-1451 $1^{14}$ tout en conservant sa charge d'écuyer et de chambellan du duc Pierre de Bretagne. La confiance accordée par le duc trouve son illustration dans une charge à l'échelon urbain. Par ailleurs, si la documentation est moins importante pour les décennies suivantes, les Lespervier conservent une place de choix dans la ville en 1500 grâce à Arthur et Charles. Le premier est d'abord grand veneur du duché avant d'être le capitaine de ville tandis que le second est à la fois son lieutenant en ville, ainsi que le tranchant de la reine Anne ${ }^{15}$. Par ailleurs, l'alliance se fait aussi matrimoniale: Arthur de Lespervier épouse la fille du trésorier du duché de Bretagne, Landais. L'alliance n'est pas ici celle de nobles, mais bien plutôt celle d'officiers qui manifestent leur importance à l'échelon administratif. L'administration propose désormais les mêmes possibilités de mobilité que le seul service des armes.

Manifeste de la fidélité au duc et à la ville, la mobilité sociale procède d'un groupe en quête d'assimilation dans la ville : Nantes, au cœur de la guerre de Bretagne, est partie prenante d'un jeu de fidélités à l'échelle de l'Occident médiéval. Les Castillans se distinguent ici particulièrement dans leur rôle au sein de la cause bretonne. Les marchands participent largement à la défense de la ville : on mentionne ainsi les «marchands de la Nation d'Espagne » comme ceux ayant fourni des balles de laine, perdues lors du siège de la ville, et à qui il est donc nécessaire d'accorder des réparations ${ }^{16}$. L'assimilation des Castillans est totale au milieu du XVI ${ }^{\mathrm{e}}$ siècle : l'un des membres de la famille Astudillo fut prêteur puis échevin de la ville. Les membres des grandes familles de

\footnotetext{
${ }_{14}^{14}$ Nantes, Archives Municipales, EE 11, $11 \mathrm{r}^{\mathrm{o}}, 12 \mathrm{r}^{\mathrm{o}}$.

${ }^{15}$ Nantes, Archives Municipales, EE 11, $21 \mathrm{r}^{\mathrm{o}}$. Le tranchant était l'écuyer de la reine chargé notamment de lui couper sa viande ou encore qui avait en garde l'étendard royal.

${ }^{16}$ Nantes, Archives Municipales, BB 1, 13, $\mathrm{r}^{\mathrm{o}}$. Les marchands castillans prennent une place de plus en plus importante dans la ville suite à l'établissement d'un consulat espagnol par les marchands de Burgos dès 1429.
} 
Burgos intègrent ainsi l'élite communale par mariage, achat de seigneurie ou octroi de privilèges économiques : ces différentes formes d'intégration peuvent être successives ou concomitantes. Une élite marchande se dessine, grâce aux Castillans, mais aussi grâce à une élite marchande locale qui fournit la majeure partie des miseurs ${ }^{17}$.

Avec ces deux exemples, la diversité des origines de cette élite est esquissée, entre seigneurs de grand lignage et petits seigneurs récents : les Lespervier n'inscrivent leur nom dans leur seigneurie que par son achat en 1448. Il semble que l'on sorte d'une hiérarchie sociale marquée par les grands barons bretons pour passer à la ville comme espace de rencontre et d'alliance d'acteurs issus de milieux différents. La transition se fait sans heurts car elle se fond plus dans le renouvellement des élites qu'elle ne s'insère dans le cadre politique. De la même manière, il semble que la quête d'autonomie municipale ne constitue pas de rupture dans le processus de rattachement du duché à la couronne de France.

\section{Quête et réalités d'une autonomie}

La ville de Nantes jouit d'une grande autonomie au sein de l'espace de la France de l'Ouest : l'administration et le commandement de la ville sont majoritairement aux mains de bourgeois formant le Conseil des Bourgeois ${ }^{18}$, dont le nombre varie, avec à leur tête à partir de

\footnotetext{
${ }^{17}$ Nantes, Archives Municipales, BB 134, $14 \mathrm{r}^{\mathrm{o}}$ et $16 \mathrm{r}^{\mathrm{o}}$. L'office de miseur est spécifique à six villes bretonnes: Nantes, Lamballe, Vitré, Quimper, Vannes et Rennes. Le miseur a la charge des comptes municipaux et ses travaux sont rassemblés dans les comptes des miseurs. Bertrand Morin est élu comme miseur, et, même une fois sorti de sa charge, des indemnités lui sont accordées à titre de récompense pour la manière dont il s'est acquitté de son office.

${ }^{18}$ Le Conseil des Bourgeois ou Conseil de ville agit au nom de la cité et est reconnu par le duc Jean V à partir de 1420. Il réunit des officiers ducaux : le sénéchal, le capitaine, le connétable, de six chanoines, ainsi que le chapitre cathédral et la collégiale Notre Dame, et enfin, comme représentants des habitants, douze bourgeois, auxquels s'ajoutent un miseur et un contrôleur chargés d'assister le procureur.
} 
1411 un procureur des bourgeois. Les bourgeois sont donc partie prenante de la gestion financière et de la défense de la ville.

Les assemblées ont lieu dans différentes maisons de la ville ${ }^{19}$ et l'itinérance du Conseil des Bourgeois dans la seconde moitié du $\mathrm{XV}^{\mathrm{e}}$ siècle témoigne de la force de l'identité urbaine : pour être reconnu, il ne nécessite nul lieu particulier pour s'affirmer. Aucun marqueur social n'est nécessaire pour témoigner de leur autorité en tant que membres de leur institution communale. Malgré tout, au service de la ville, chaque acteur de la ville peut avoir plusieurs visages et ne pas être cloisonné à sa fonction.

\section{Autonomie et cumul des charges et offices}

Chacun cherche sa place dans cette ville de la fin $d u X V^{e}$ siècle, chacun cherche son rôle, et les fonctions se multiplient, des plus visibles comme celle de trompette, héraut de la ville ${ }^{20}$, à celles supposées compléter un revenu : le nombre de portiers de la ville issus de lignages reconnus augmente, alors même que d'après les quittances, la fonction parait plus honorifique que rémunératrice.

La charge peut, par ailleurs, être facilement déléguée, ce qui facilite leur cumul. Jean du Besic en est une illustration parlante: en 1518 , il est à la fois valet de pied et sommelier de panneterie ${ }^{21}$ de la reine Claude de France, ainsi que portier de la porte Poissonnière en ville. La liberté vis-à-vis de cette dernière charge est réelle : il afferme pour un an

\footnotetext{
${ }^{19}$ Nantes, Archives Municipales, BB 1, 7, ro . La maison des Thomas est prêtée plusieurs semaines par an, entre 1450 et 1480 .

${ }^{20}$ Nantes, Archives Municipales, BB 137, 1 à $18\left(\mathrm{r}^{\mathrm{o}}\right)$. Quittances de Denis Guillard, le premier trompette nommé de la ville de Nantes, héraut municipal de 1487 à 1503. Deux autres trompettes seulement lui succèdent de 1517 à 1548, Jamet le Pin et Pierre la Plume.

${ }^{21}$ Le sommelier de panneterie est sous les ordres du Grand Panetier et s'occupe de la table de la reine: il est chargé d'y placer la nef contenant salière, serviettes et couteaux.
} 
son devoir à cette charge à un archer pour 16 livres 6 sous 8 derniers ${ }^{22}$. Pour autant, il conserve sa charge 33 ans comme le souligne une étude de ses quittances : elles renseignent sur une somme constante sur toute la période de 72 sols par quartier de service ${ }^{23}$.

Le grand nombre ainsi que la diversité des quittances produites par les miseurs de la ville témoigne de la vigueur des charges et des offices municipaux. Être titulaire d'un office ou d'une responsabilité dans la ville garantit une respectabilité aussi reconnue que le serait un office auprès de la duchesse-reine par exemple. L'étude de ces phénomènes traduit donc des transformations progressives dans les modalités d'ascension sociale.

\section{De la «nécessité de la ville» à la constitution d'une élite artisanale}

Les circonstances de la Guerre de Bretagne entraînent une augmentation des offices : on assiste à la constitution de ce qui pourrait être qualifié d'élite artisanale, qui reçoit des commandes du clerc de l'artillerie ${ }^{24}$. Par ailleurs, le modèle de commandes réalisées par la ville s'uniformise : un certain nombre de fondeurs travaillent exclusivement pour la ville de Nantes. Colin Rivière fond ainsi un certain nombre de bâtons pour la défense de la ville, et le paiement n'est réclamé qu'après la réalisation : l'absence de mention de la somme dans l'acte, et la référence à l'ancien miseur signalent que, si la commande a été faite par la ville, la

\footnotetext{
${ }^{22}$ Nantes, Archives Municipales, EE 13, $55 \mathrm{r}^{\circ}$.

${ }^{23}$ Nantes, Archives Municipales, EE 13, 57-73, $\mathrm{r}^{\mathrm{O}}$.

${ }^{24}$ Le clerc de l'artillerie est lui-même sous les ordres d'un grand maître de l'artillerie, mais reste chargé de la majeure partie du volet économique et de contrôle des approvisionnements. Nantes, Archives Municipales, EE 159, 9, $\mathrm{r}^{\mathrm{o}}$. Jehan Chausse clerc de l'artillerie en 1489 tient les comptes et est chargé de s'assurer du bon approvisionnement des fondeurs chargés de réaliser les canons de la ville. Les comptes se présentent sous la forme de cahiers à l'intérieur desquels le compte est réalisé de manière thématique. On distingue en 1489 dans les cahiers de comptes du clerc de l'artillerie les dépenses générales relatives à l'état de guerre, puis celles liées aux pierres, au salpêtre, au charbon, aux poudres.
} 
rétribution n'en était pas définie par avance ${ }^{25}$. Il semble aussi que, dans la plupart des cas, le métal était fourni par la ville elle-même ${ }^{26}$. Ces remarques témoignent donc du nouveau rapport de dépendance établi entre les métiers liés à l'artillerie et la ville, le duc ou le roi. En effet, face à la nécessité de la guerre, les métiers d'artillerie peuvent être la porte d'entrée à des charges prestigieuses : il faut noter que l'artillerie est indépendante de l'armée ducale en tant que telle. Le duc ou roi est donc dépendant à la fois de la manufacture nantaise, et, dans une certaine mesure, de la décision des bourgeois de la ville forte qu'est Nantes. L'office de clerc de l'artillerie est donc au cœur des tractations militaroéconomiques entre la ville et le roi. Il fait en effet partie des élites nantaises, car il est en mesure de manipuler l'argent.

Il nous semble toutefois nécessaire de noter que l'illustration la plus notable de l'importance de cette élite artisanale est à chercher dans les sources concernant le siège de la ville par les Français du 20 juin au 6 août 1487. Ceux que nous avons définis comme faisant partie de cette élite artisanale participent directement à l'administration de la ville, et plus seulement à sa défense, en tant qu'il faut « des gens suffizans pour la [tuicion de ceste ville] faire, et résister à la mauvaise et damnable volonté desdits Françoiz $^{27} »$. De ces «gens suffizans» que sont les clercs d'artillerie peuvent émerger des figures de la ville, à l'instar de Gerardin

\footnotetext{
25 Nantes, Archives Municipales, EE 156, $10 \mathrm{r}^{\mathrm{o}}$. On remarque plusieurs autres exemples de ce type, notamment des réclamations faites par Jean Challon pour d'autres bâtons (canons) de fonte nécessaires à la défense de la ville, tels que le Donatien.

${ }^{26}$ Nantes, Archives Municipales, EE 156, $16 \mathrm{r}^{\mathrm{o}}$; EE 156, $17 \mathrm{r}^{\mathrm{o}}$ : Pierre Foutin et Alain Gabard reçoivent deux mortiers de la part du duc afin d'en faire des canons, le Cousin et le Maréchal, mais cela n'a pas suffi, et ils demandent réparation pour le cuivre qu'ils ont dû eux-mêmes fournir. Anecdotique, certes, mais la valeur accordée au métal en tant que tel est au cœur des échanges entre le pouvoir ducal et sa ville : après la bataille de Saint Aubin du Cormier en 1488, beaucoup de métal est donné par la duchesse à la ville, par l'entremise du garde de l'artillerie, pour remplacer les canons d'arquebuse perdus par la ville lors de la bataille.

${ }^{27}$ Nantes, Archives Municipales, EE 186 r $^{\circ}$.
} 
de Billy: clerc de l'artillerie en 1489, il est chargé en 1493 par l'assemblée de ville de se rendre auprès du roi afin de demander que le conseil et la chancellerie de Bretagne se tiennent à Nantes ${ }^{28}$. Les nécessités de la ville favorisent donc la reconnaissance d'individualités particulières. Désormais, la qualité professionnelle produit de la reconnaissance : le métier des armes n'est plus le seul à participer à la réputation. Être artilleur, ou simplement participer à l'effort de la ville pendant le siège attire désormais les faveurs de la commune.

\section{Élite et autonomie économique}

Le dynamisme de l'élite communale conduit le duc à céder à la ville un certain nombre de droits, le principal étant celui de battre monnaie. De plus, les habitants de Nantes obtiennent entre 1487 et 1489 le droit de seigneuriage sur les monnaies, à savoir le droit de disposer du profit dégagé sur l'activité de frappe monétaire grâce à la valeur de la monnaie dans les échanges commerciaux. Ce droit était auparavant détenu par les ducs de Bretagne: la ville acquiert une indépendance et une liberté qui facilitent les travaux publics ${ }^{29}$. Le droit de seigneuriage perçu doit néanmoins être utilisé exclusivement pour les réparations et fortifications de la ville ${ }^{30}$. On pourrait qualifier ce droit d'impôt indirect, ou impôt pris sur la source même, à savoir la monnaie. Enfin, en 1488, les Nantais obtiennent le droit de nommer leurs monnayeurs et d'en recevoir leur serment ${ }^{31}$. Ce premier octroi d'autonomie économique souligne ainsi qu'il n'entre pas en contradiction avec la constitution, ou plutôt l'intégration dans un État moderne. Le recours à ces privilèges

\footnotetext{
${ }^{28}$ Nantes, Archives Municipales, BB $18, \mathrm{r}^{\mathrm{o}}$ et $\mathrm{v}^{\mathrm{o}}$.

29 Nantes, Archives Municipales, HH 24, $1 \mathrm{r}^{\mathrm{o}}$. La perception du seigneuriage correspond à 500 marcs d'argent.

${ }^{30}$ Le réemploi de l'octroi participe aussi de ces travaux publics.

${ }^{31}$ Nantes, Archives Municipales, HH 24, 2, $\mathrm{r}^{\circ}$.
} 
économiques favorise ainsi l'autonomisation des acteurs économiques de la ville.

L'autonomie économique n'est pas seulement accordée aux institutions communales. La liberté laissée au clergé par le duc Jean en 1440 quant au droit d'apétissement sur le $\operatorname{vin}^{32}$ n'est rendue possible que par l'obligation faite d'en utiliser la somme pour les travaux de la cathédrale et plus particulièrement du portail Saint-Pierre ${ }^{33}$. La construction et l'embellissement de la cathédrale font donc partie d'une véritable politique communale: la notion de commande publique se précise. L'argent issu du chapitre est réemployé dans une œuvre du chapitre, alors que les devoirs issus de vins produits dans d'autres propriétés ecclésiastiques sont employés plus largement aux réparations de la ville ${ }^{34}$. Il semble ainsi que l'on assiste à une prise de conscience de l'espace public : la ville est désormais considérée dans son ensemble, et l'espace communal n'est plus seulement un espace juridictionnel. Si la ville prend des droits sur l'espace, l'élite communale s'arroge ainsi l'espace public ${ }^{35}$.

Dans cette longue séquence de constructions, la «nécessité de la ville » est le prétexte à l'affirmation d'institutions dont la force réside dans la liberté économique qui leur est accordée.

L'argent ainsi que la nécessité de la ville favorisent l'affirmation de cette élite, qu'elle soit laïque ou religieuse. L'autonomie et

\footnotetext{
${ }^{32}$ Le devoir d'apétissement est parfois appelé droit de billot: il s'agit d'un impôt devant être utilisé pour des ouvrages publics. Les gens du chapitre et autres gens d'Église ont ainsi le droit de vendre leur vin au détail dans des franchises selon la mesure d'apétissement.

33 Nantes, Archives Municipales, GG 600, $1 \mathrm{r}^{\mathrm{o}}$. Le document mentionne que les devoirs perçus de l'apétissement du vin produit sur les fiefs du clergé devront être employés à la confection et à la réparation du portail Saint Pierre.

${ }^{34}$ Nantes, Archives Municipales, GG 600, $2 \mathrm{r}^{\mathrm{o}}$.

${ }^{35}$ L'Espace public au Moyen Âge : débats autour de Jürgen Habermas, dir. Patrick Boucheron et Nicolas Offensdadt, Paris, PUF, coll. «Le Nœud gordien », 2011.
} 
l'indépendance des élites urbaines s'étendent à tout l'espace urbain, et pas seulement aux seules propriétés communales. Ce processus s'opérant de part et d'autre de la fin du Moyen Âge, il inclut donc une dimension à la fois spatiale et chronologique.

Nantes, et plus largement la Bretagne s'inscrivent dans un Moyen Âge qui ne finit pas : entre la figure sublimée d'Anne de Bretagne et l'évocation dans plusieurs rencontres scientifiques de «Nantes la flamboyante », s'attacher à ceux qui ont donné à la ville son éclat et servi la reine et duchesse permet ainsi de rejeter l'idée d'un Moyen Âge finissant. Une identité urbaine se définit, qui n'est celle ni des nobles ni de chevaliers, mais plutôt celle d'officiers qui accèdent à la seigneurie grâce à leur charge. Pour autant, on ne peut pas parler d'ordre nouveau : ce n'est ni un Tiers État avant l'heure, ni une quelconque tentative de la bourgeoisie d'accéder à la noblesse de robe. Le Moyen Âge ne finit pas avec une coupure nette, au sens où le véritable renversement n'aura lieu que dans la crise de cette petite et moyenne noblesse à la toute fin du $\mathrm{XVI}^{\mathrm{e}}$ siècle $^{36}$ : on remarque «une série de petits déchirements partiels ${ }^{37}$ » qui participent du renouvellement d'une élite urbaine qui se définit différemment et n'investit plus tout à fait de la même manière l'espace. Il n'y a ainsi pas de bouleversement des codes sociaux, mais bien plutôt des changements à la marge qui rognent sur la classe dominante. La ville de Nantes réussit à conserver la puissance administrative conférée par la constitution d'un État breton, tout en étant perméable aux crises et changements de la société : la ville de la fin du $\mathrm{Xv}^{\mathrm{e}}$ siècle incarne une transition, plus qu'une rupture, et l'élite urbaine reste le produit de la continuité entre un État breton finissant et une province naissante.

\footnotetext{
${ }^{36}$ Michel Nassiet, Noblesse et pauvreté, op. cit.

${ }^{37}$ Paul Zumthor, Parler du Moyen Âge, Paris, Éditions de Minuit, coll. «Critique », 1980.
} 